\title{
Anaesthesia for thoracic surgery: recent advances
}

Jonathan L. Benumof MD

This refresher course is in three sections. The first, "correct positioning of double-lumen tube," reviews what is often the most important determinant as to whether thoracic cases (in particular one-lung ventilation cases) proceed smoothly. If the double-lumen tube is in the correct position, the nondependent lung will collapse completely and easily, the surgeon will be able to work efficiently without damaging the lung, and the dependent lung will be unobstructed and easy to ventilate. The second section, "determinants of arterial oxygenation and intrapulmonary shunt during one-lung ventilation," describes the distribution of blood flow during one-lung ventilation and the factors that may alter that distribution. The choice of anaesthetic drug and technique are factors that may influence the distribution of blood flow during one-lung ventilation. The third section, "management of one-lung ventilation," outlines the initial management of onelung ventilation and what to do if hypoxaemia ensues.

\section{Correct positioning of double-lumen tube}

When surgery is performed on the right lung a left-sided double-lumen tube (DLT) is used (Figure 1). When surgery of the left lung is performed (Figure 1), either a left- or right-sided DLT may be used. However, use of a right-sided DLT for left lung surgery introduces the risk of inadequate ventilation of the right upper lobe (RUL) if the RUL ventilation slot is not closely opposed to the RUL orifice (see Figures 1 and 5). To avoid this complication some anaesthetists use a left sided DLT for all cases requiring one-lung ventilation. If clamping of the left mainstem bronchus is necessary, the DLT can be withdrawn into the trachea at that time (after deflating the cuffs) and then used as a single-lumen tube (deflate only the left lumen cuff and use both lumens to ventilate the right lung) (Figure 1). We use a left lung, clear, nontoxic, plastic, low pressure cuff, disposable Robertshaw type DLT.
The Robertshaw type DLT is passed with the distal curvature initially concave anteriorly (Figure 2, panel 1). After the tube tip passes the larynx the tube is rotated 90 degrees so that the proximal curve is concave anteriorly (Figure 2, panel 2) to allow
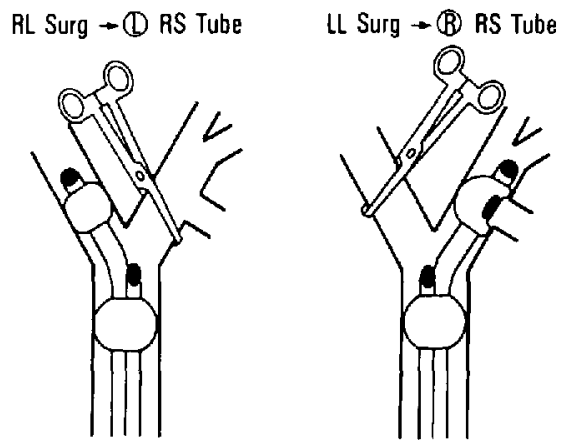

LL Surg $\rightarrow$ (D) RS Tube Pulled Back

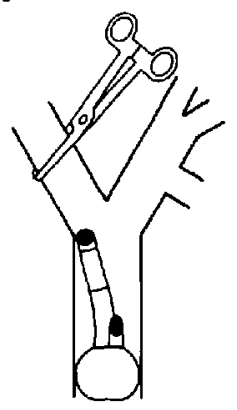

FIGURE 1 Position of left and right-sided double-lumen tubes. See text for details.

Department of Anesthesiology, University of California, San Diego, Anesthesia Research Laboratory, T-001, La Jolla, California, 92093, U.S.A. 


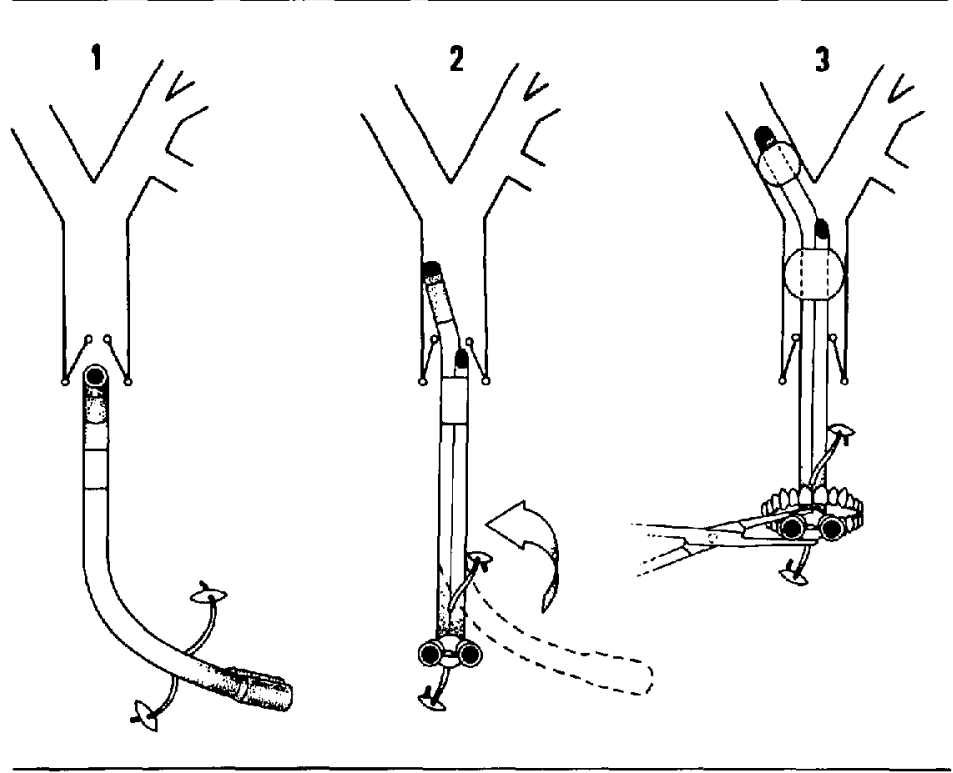

FIGURE 2 Positioning of left-sided double-lumen tube during insertion (panels 1, 2). confirmation of correct placement (panel 3). See text for details.

endobronchial intubation on the appropriate side. The tube is then inserted until most of it is in (common two-lumen moulding is usually at the level of the teeth) and/or a moderate resistance to further passage in encountered. Once the tube tip is thought to be endobronchial, both the tracheal and endobronchial cuffs should be inflated and bilateral ventilation checked. Then, one connecting tube should be clamped (Figure 2, panel 3) and the disappearance of breath sounds on that side and their continuation on the other side should be listened for to ensure the proper tube position.

If the position of the DLT is correct, then the anaesthetist should be able to make four observations during unilateral clamping. First, the breath sounds should only be heard on the contralateral side. Second, the contralateral lung should feel reasonably compliant. Third, the breath sounds should disappear on the ipsilateral (clamped) side. Fourth, only the contralateral chest should rise and fall with ventilation, giving the chest a "rocking boat" motion.

There are three possible malpositions for a DLT (Figure 3). It may be inserted too far into the left or the right mainstem bronchus, or it may be inserted too little so that both lumens are in the trachea. In each malposition the left cuff, when inflated, blocks the right lumen and this can be taken advantage of to diagnose the DLT malposition. When the left cuff is inflated and the left lumen is clamped (ventilation is only through the right lumen), the left cuff will block the right lumen in all three malpositions and the breath sounds will be either absent or very diminished. When the left cuff is then deflated, breath sounds will only be heard on the left side when the DLT is in too far on the left; breath sounds will be heard on both sides when the DLT is out too far; and breath sounds will only be heard in the right lung when the DLT is in too far on the right.

Unfortunately, the above scheme of left lumen clamping, left cuff inflation-deflation, and auscultation manoeuvres to determine DLT malposition is not useable and/or reliable all the time. The reasons for failure are: (1) once the patient is prepared and draped, the chest is no longer available for auscultation; (2) use of breath sounds as an endpoint is often not sensitive enough because of either preexisting or anaesthesia-induced lung disease; (3) the tube may move during the operation (surgical traction, patient coughing); (4) the tube may be just barely 


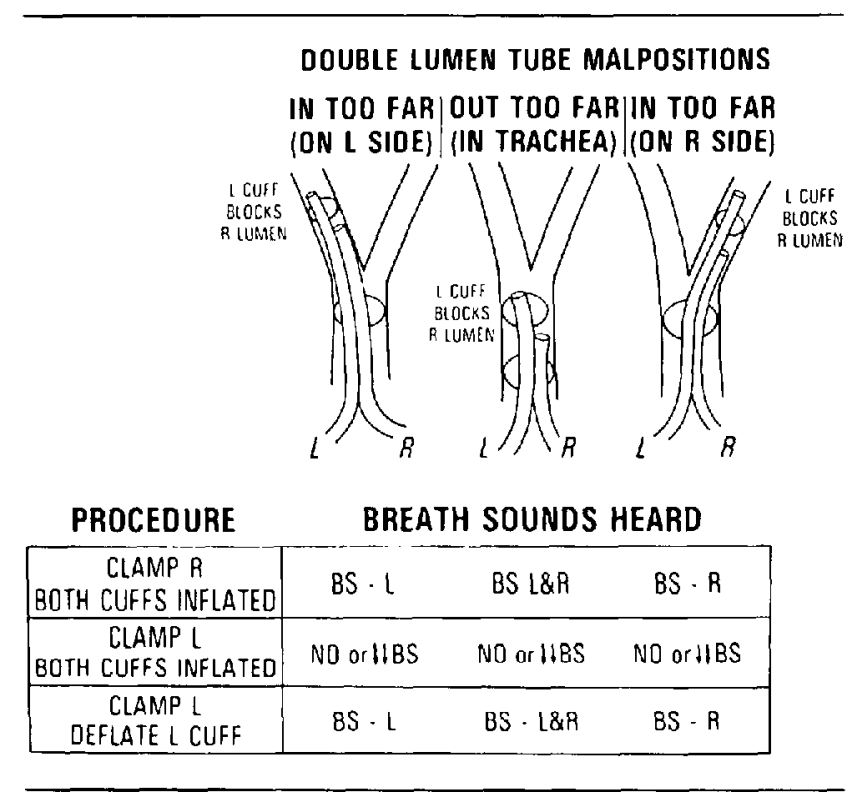

FIGURE 3 Methods to detect malpositioning of double lumen tubes.

DOUBLE LUMEN TUBE POSITION AND CUFF SEAL:

FIBEROPTIC BRONCHOSCOPE

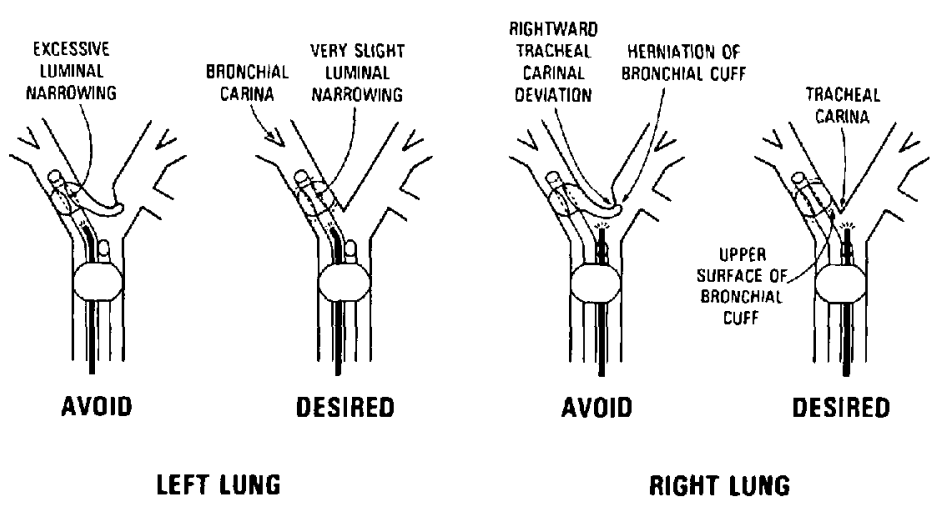

FIGURE 4 Use of fibcroptic bronchoscope to confirm correct double-lumen tube positioning and cuff seal. 


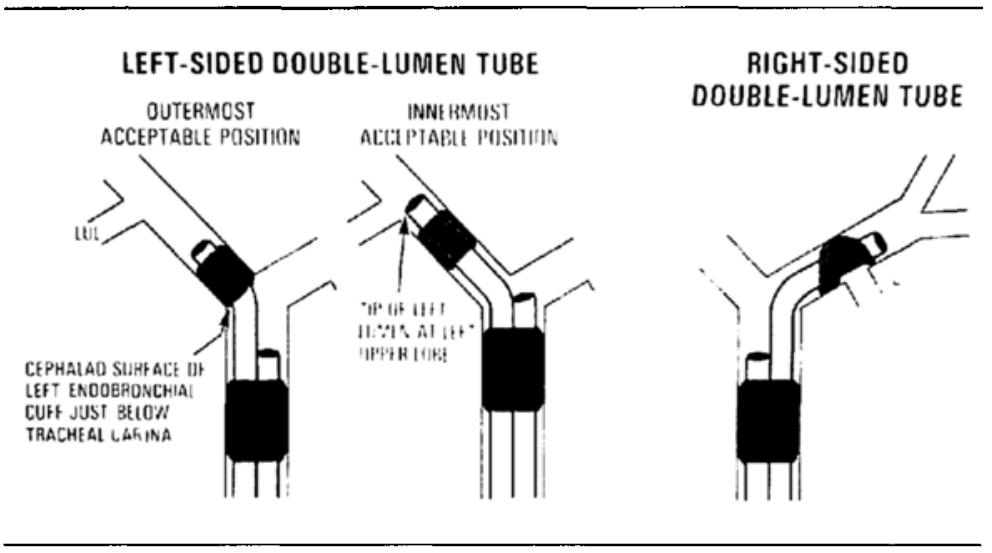

FIGURE 5 Position of double-lumen tubes in relation to carina and upper lobe bronchi.

malpositioned; and (5) some combination of the above.

Any doubt in the position of the DLT may be resolved by the use of fiberoptic bronchoscopy (FOB). A paediatric FOB (outside diameter less than $4.5 \mathrm{~mm}$ ) can be passed down the lumens of all sized $(35,37,39,41$ French) DLTs. A $4.9 \mathrm{~mm}$ outside diameter FOB will not pass down the 35 French and is a tight squeeze through a 37 French tube. The simplest method of determining DLT position is to pass the FOB down the right lumen.

Looking down the right lumen, the endoscopist should see a clear straightahead view of the tracheal carina and the upper surface of the left endobronchial cuff (blue for the National Catheter Corp. DLT) just below the tracheal carina (Figure 4, two right panels). Looking down the right lumen, the endoscopist should not see the left cuff herniating over the carina or the carina pushed over to the right and compromising the right mainstem bronchial orifice (Figure 4, two right panels). Looking down the left lumen (which is not usually done), the endoscopist should see a clear straightahead view of the bronchial carina and only a slight left-luminal narrowing (Figure 4, two left panels). I find that I am doubtful of the position of the DLT (and therefore use the FOB) in about two out of ten patients in the supine position (when the tube first goes in) and in an additional one of the remaining eight when the patient is turned into the lateral decubitus position. In addition, the FOB is used anytime during the case there is a doubt about position of the DLT.
The importance of seeing the upper surface of the left endobronchial cuff below the tracheal carina needs to be emphasized. ${ }^{2}$ The outermost acceptable position of a left-sided DLT occurs when the left endobronchial cuff is just below the tracheal carina. If a left-sided DLT is pulled out any further, the left endobronchial cuff will obstruct the trachea and the right mainstem bronchus (Figure 4, left panel). The innermost acceptable position occurs when the distal tip of the left lumen is at the left upper lobe (LUL) bronchus, because further insertion will obstruct the LUL (Figure 5, middle panel). The distance between the right and left lumen tips for the National Catheter Corp. tube $(=70 \mathrm{~mm})$ is longer than the length of the left mainstem bronchus ( $=50 \mathrm{~mm}$ in adult males and females). Thus, it is possible for the right lumen to be above the tracheal carina while the left lumen tip obstructs the LUL. The distance between the upper surface of the left endobronchial balloon and the left tip is $25 \mathrm{~mm}$. Thus, when the upper surface of the left endobronchial balloon is just below the tracheal carina, it is not possible for the left tip to obstruct the LUL.

Determinants of arterial oxygenation and intrapulmonary shunt during one lung ventilation ${ }^{3,4}$

\section{Distribution of blood flow during two-lung} ventilation and lateral decubitus position

The fractional blood flow to the left and right (and larger) lungs in the upright and supine positions is 45 and 55 per cent respectively. However, one-lung ventilation is usually performed in the lateral 


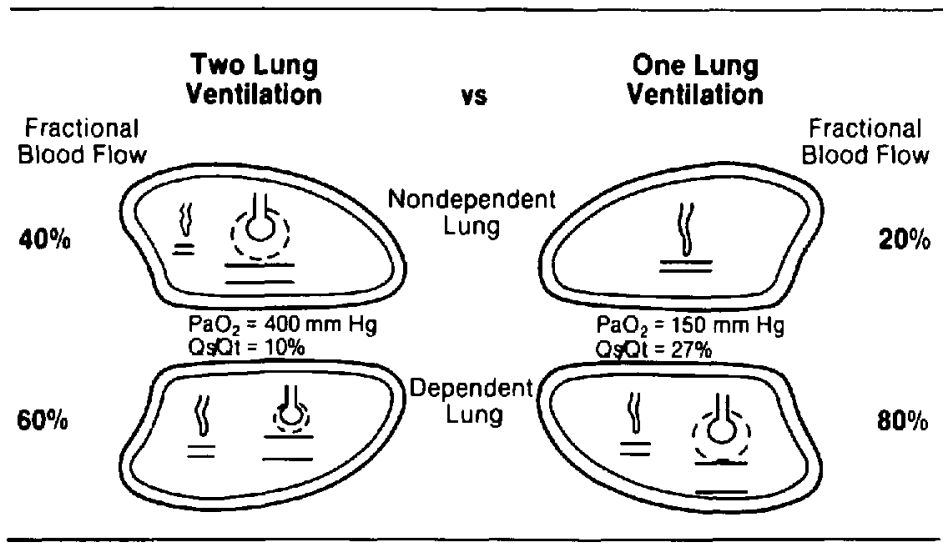

FIGURE 6 Fractional blood flow and resultant $\mathrm{PaO}_{2}$ and $\dot{\mathrm{Q}}_{\mathrm{s}} / \dot{\mathrm{Q}}_{1}$ during two-lung ventilation and one-lung ventilation.

decubitus position and gravitational forces will alter the distribution of blood flow between the two lungs (from the supine distribution) so that the nondependent lung receives relatively less and the dependent lung receives relatively more blood flow. Thus, when the left lung is the nondependent lung the fractional blood flow between the left and right lungs is 35 and 65 per cent respectively, and when the right lung is the nondependent lung the fractional blood flow between the left and right lung is 45 and 55 per cent respectively.

The left hand side of Figure 6 shows typical two-lung ventilation conditions in the lateral decubitus position for a typical pulmonary resection patient with mild-moderate lung disease (smoking history). The average nondependent and dependent lung blood flow distribution would be 40 per cent $(35+45 / 2)$ and 60 per cent $(65+55 / 2)$, respectively. The patient has a ten per cent $\dot{Q}_{s} / Q_{t}$ on two-lung ventilation which is assumed to be distributed equally between the two lungs, and the $\mathrm{PaO}_{2}$ is $400 \mathrm{mmHg}$ on an $\mathrm{FIO}_{2}=1.0$.

\section{Distribution of blood flow during one-lung ventilation, lateral decubitus position}

When one-lung ventilation is employed the nondependent lung is the nonventilated and collapsed lung and the dependent lung is the ventilated lung. Consequently, one-lung ventilation creates an obligatory shunt through the nonventilated lung which is not present during two-lung ventilation. Thus, it is not surprising that, for the same $\mathrm{FIO}_{2}$ and haemodynamic and metabolic status, one-lung ventilation results in a much decreased $\mathrm{PaO}_{2}$. However, and fortunately, there are both passive mechanical and active vasoconstrictor mechanisms that are usually operant that minimize the blood flow to the nonventilated lung and thereby prevent the $\mathrm{PaO}_{2}$ from decreasing as much as might be expected.

\section{BLOOD FLOW TO THE NONDEPENDENT \\ NONYENTILATED LUNG}

The passive mechanical mechanisms decreasing blood flow to the nondependent lung consist of gravity and surgical interference with blood flow. Gravity contributes to the vertical gradient in the distribution of pulmonary blood flow during onelung ventilation in the lateral decubitus position just as it does during two-lung ventilation in the lateral decubitus position. The gravity component of blood flow reduction to the nondependent lung should be constant with respect to both time and magnitude. The surgical interference of nondependent lung blood flow involves compression and retraction of the nondependent lung and ligation of nondependent lung blood vessels. The surgical interference component of nondependent lung blood flow reduction should be variable with respect to both time and magnitude.

The most significant reduction in blood flow to the nondependent lung is caused by an active vasoconstrictor mechanism. The normal response of the pulmonary vasculature to atelectasis is an increase 


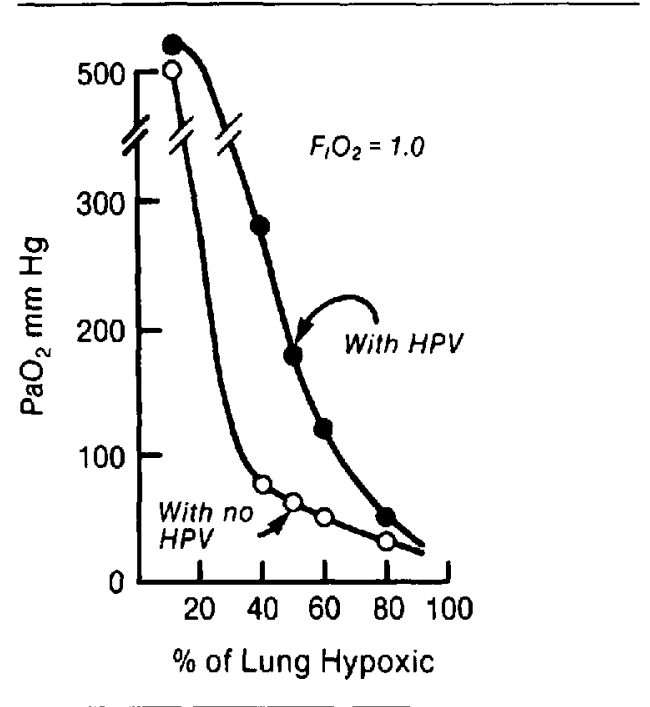

FIGURE 7 Effect of hypoxic pulmonary vasoconstriction (HPV).

in pulmonary vascular resistance (PVR) (in the atelectatic lung) and the increase in atelectatic lung PVR is thought to be due almost entirely to hypoxic pulmonary vasoconstriction (HPV). This conclusion applies whether ventilation is spontaneous or with positive pressure, and whether the chest is open or closed. It is possible that some further subacute (greater than 30 minutes) decrease in blood flow to the atelectatic lung is due to mechanical effects of atelectasis on lung blood vessels.

The selective increase in atelectatic lung PVR diverts blood flow away from the atelectatic lung towards the remaining normoxic or hyperoxic ventilated lung. The diversion of blood flow minimizes the amount of shunt flow that occurs through hypoxic lung. Figure 7 shows the theoretically expected effect of HPV on $\mathrm{PaO}_{2}$ as the amount of lung that is made hypoxic increases. When very little of the lung is hypoxic (near zero per cent) it does not matter, in terms of $\mathrm{PaO}_{2}$, whether the small amount of lung has HPV or not because in either case the shunt will be small. When most of the lung is hypoxic (near 100 per cent) there is no significant normoxic region for the hypoxic region to divert flow to and again it does not matter, in terms of $\mathrm{PaO}_{2}$, whether the hypoxic region has HPV or not. When the amount of lung that is hypoxic is intermediate (between 30-70 per cent), which is the amount of lung that is hypoxic during the one-lung ventilation condition, there is a large difference between the $\mathrm{PaO}_{2}$ expected with a normal amount of HPV compared to when there is no HPV. In fact, in this range of hypoxic lung, HPV can increase $\mathrm{PaO}_{2}$ from levels that might cause arrhythmias to much higher and safer values. It is not surprising then, that numerous clinical one-lung ventilation studies have found that the shunt through the nonventilated lung is usually $20-25$ per cent of the cardiac output as opposed to the $35-45$ per cent shunt that might be expected if there was no HPV in the nonventilated lung. Thus, HPV is an autoregulatory mechanism that protects the $\mathrm{PaO}_{2}$ by decreasing the amount of shunt flow that can occur through hypoxic lung.

The right side of Figure 6 shows what happens to fractional lung blood flows, $\dot{\mathrm{Q}}_{\mathrm{s}} / \dot{\mathrm{Q}}_{1}$ and $\mathrm{PaO}_{2}$ when the typical pulmonary resection patient with mildmoderate lung disease is changed from two-lung ventilation to one-lung ventilation in the lateral decubitus position and gravity and HPV are the only forces affecting the one-lung ventilation blood fow distribution. Since HPV can decrease blood flow to a single lung by 50 per cent, the 35 per cent fractional flow to the nondependent lung that can participate in HPV (five per cent nondependent lung fractional blood flow was already $\dot{\mathrm{Q}}_{\mathrm{s}} / \dot{\mathrm{Q}}_{\mathrm{t}}$ flow) will be decreased to 17 per cent. Thus, the fractional nondependent lung blood flow decreases from 40 per cent to 22 per cent ( 17 per cent +5 per cent), total $\dot{Q}_{s} / \dot{Q}_{1}$ increases from 10 per cent to 27 per cent (22 per cent in the nondependent lung and five per cent in the dependent lung) and $\mathrm{PaO}_{2}$ decreases from $400 \mathrm{mmHg}$ to $150 \mathrm{mmHg}$. However, one-lung ventilation $\dot{Q}_{S} / \dot{Q}_{t}$ may be higher if the dependent lung develops a larger shunt compartment (see below).

Anaesthesia and surgery involve numerous factors that may decrease the amount of nondependent lung HPV blood flow diversion. They can be divided into physiological, drug, and nondependent and dependent lung disease factors. The physiological factors, such as pulmonary vascular pressure, $\dot{\mathrm{Q}}_{i}$, $\mathrm{PvO}_{2}$, and $\mathrm{PaCO}_{2}$ must be significantly changed from normal in order to have a major inhibitory effect on nondependent lung HPV flow diversion. Changes of this magnitude in these variables represent a major disturbance to homeostasis and can be 
considered life-threatening. As such they require immediate treatment and their effect on $\mathrm{PaO}_{2}$ during one-lung ventilation, although clearly demonstrable experimentally, must be regarded as a secondary clinical consideration and therefore will not be discussed here.

Various drugs used in clinical anaesthesia can inhibit HPV flow diversion. Most systematic vasodilator drugs inhibit regional HPV. Those specifically studied include nitroglycerin, nitroprusside, dobutamine, several calcium antagonists, and many beta-2-agonists (isoproterenol, ritodrine, orciprenaline, salbutamol, ATP, and glucagon). Aminophylline and hydrazaline may not decrease HPV.

Vasoconstrictor drugs (dopamine, epinephrine, phenylephrine) seem to constrict normoxic lung vessels preferentially, thereby increasing normoxic lung PVR disproportionately. The increase in normoxic lung PVR will decrease normoxic lung blood flow and increase atelectatic lung blood flow. The effect of vasoconstrictor drugs is similar to decreasing normoxic lung $\mathrm{F}_{1} \mathrm{O}_{2}$ (see below).

Anaesthetic drugs may inhibit HPV. Many experimental studies in animals indicate that the halogenated inhalational anaesthetics exhibit a dose dependent inhibition of regional HPV whereas the intravenous anaesthetics do not. However, recent studies in patients undergoing thoractomy in the lateral decubitus position indicate that halothane and isoflurane, in approximately $1 \mathrm{MAC}$ doses, do not decrease $\mathrm{PaO}_{2}$ any more than intravenous anaesthesia during stable one-lung ventilation conditions. ${ }^{5,6}$ Although these studies did not examine whether HPV in the nondependent lung was inhibited or not, the results were consistent with the contention that these drugs did not have a major inhibitory effect on nondependent lung HPV. Since these drugs have a number of desirable properties (permit the use of a higher $\mathrm{FiO}_{2}$, are rapidly eliminated, have a salutory effect on bronchomotor tone, and have few negative cardiovascular effects at a $1 \mathrm{MAC}$ dose), and do not decrease $\mathrm{PaO}_{2}$ any more than intravenous anaesthesia during human one-lung ventilation conditions, they are desirable anaesthetics to use during one-lung ventilation.

The amount of disease in the nondependent lung should also be a significant determinant of the amount of blood flow to the nondependent lung. If the nondependent lung is severely diseased, then there may be a fixed reduction in blood flow to this lung preoperatively and collapse of such a diseased lung may not cause an increase in shunt, whereas collapse of a normal lung with normal blood flow may be associated with a higher nonventilated nondependent lung blood flow and shunt. A higher one-lung ventilation shunt through the nondependent lung may be more likely to occur in patients who require thoracotomy for nonpulmonary disease. However, the above theoretical relationship between the amount of nondependent lung disease and shunt during one-lung ventilation has not been studied systematically.

\section{BLOOD FLOW TO THE DEPENDENT VENTILATED}

LUNG

The dependent lung usually has an increased amount of blood flow due to both passive gravitational effects and active nondependent lung HPV. However, the dependent lung may also develop a larger hypoxic compartment intraoperatively, for several reasons. First, the dependent lung usually has a reduced lung volume due to the combined factors of induction of general anaesthesia and circumferential (and perhaps severe) compression by the mediastinum (M) from above, by the abdominal contents pressing against the diaphragm (D) from the caudad side, and by suboptimal positioning effects (P) (rolls, packs, shoulder supports) pushing in from the dependent side and axilla (Figure 8, upper left panel). Second, absorption atelectasis can also occur in regions of the lung that have low $\dot{V} / \dot{Q}$ ratios when they are exposed to high $\mathrm{FiO}_{2}$. Third, difficulty in removal of secretions may cause the development of low $\dot{V} / \dot{Q}$ and atelectatic areas in the dependent lung. Finally, maintaining the lateral decubitus position for prolonged periods may cause fluid to transudate into the dependent lung (which may be vertically below the atrium), and cause a further decrease in dependent lung volume. The development of low $\dot{\mathrm{V}} / \dot{Q}$ and/or atelectatic areas in the dependent lung will increase dependent lung PVR, thereby decreasing dependent lung blood flow and increasing nondependent lung blood flow.

\section{Management of one-lung ventilation ${ }^{7-9}$}

Conventional management of one-lung ventilation. The proper management of one-lung ventilation is based on the pathophysiology described above. First, although the theoretical possibilities of ab- 


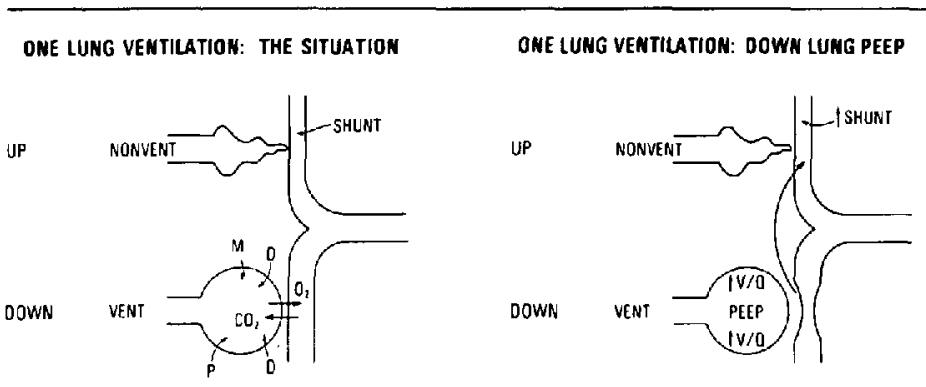

ONE LUHG VENTILATION: UP LUNG CPAP

UP

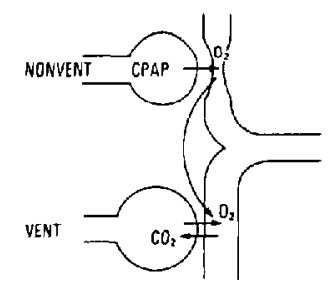

DNE LUNG VENTILATION: DIFFERENTIAL LUNG $\frac{\text { CPAP }}{\text { PEEP }}$

UP

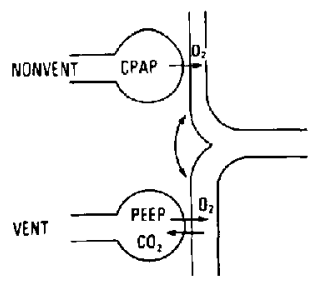

FIGURE 8 Manoeuvrcs to optimize oxygenation during one-lung ventilation. Sec text for details.

sorption atelectasis and oxygen toxicity exist, the benefits of ventilating the dependent lung with 100 per cent oxygen exceed the risks. A high $\mathrm{F}_{1} \mathrm{O}_{2}$ in the single ventilated lung may increase the $\mathrm{PaO}_{2}$ from arrhythmogenic and life-threatening levels to safer levels. In addition, a high $\mathrm{FrO}_{2}$ in the dependent lung will vasodilate the dependent lung, thereby increasing the dependent lung capability of accepting blood flow redistribution due to nondependent lung HPV. Direct oxygen toxicity will not occur during the span of the operative period and absorption atelectasis in the dependent lung is unlikely to occur in view of the remaining one-lung ventilation management characteristics outlined below. Second, the dependent lung should be ventilated with a tidal volume of $10 \mathrm{ml} \cdot \mathrm{kg}^{-1}$. Use of a tidal volume less than $10 \mathrm{ml} \cdot \mathrm{kg}^{-1}$ might promote dependent lung atelectasis. Use of a tidal volume greater than $10 \mathrm{ml} \cdot \mathrm{kg}^{-1}$ might increase dependent lung airway pressure and PVR, and thereby increase independent lung blood flow (decrease nondependent lung HPV). Third, the respiratory rate should be set so that the $\mathrm{PaCO}_{2}$ remains at $40 \mathrm{mmHg}$ (usually requires a 20 per cent increase above the rate used for two-lung ventilation). Hypocapnia should be avoided because the increase in airway pressure in the dependent lung necessary to produce hyperventilation may increase dependent lung PVR. Furthermore, hypocapnia may inhibit HPV in the nondependent lung. Finally, zero or a low level of dependent lung PEEP (less than $5 \mathrm{~cm} \mathrm{H}_{2} \mathrm{O}$ ) should be used initially because of concern of unnecessarily increasing dependent lung PVR (see selective dependent lung PEEP section below).

Occasional ventilation of the nondependent lung (one breath every five to ten minutes) causes some oxygen or oxygen-enriched gas to remain in the nonventilated lung and blood flowing through this lung can continue to take up some oxygen for a period of some minutes. Of course the effect on $\mathrm{PaO}_{2}$ of an ocasional positive pressure breath to the nondependent lung will be uncertain with regard to magnitude and temporal profile, and requires the nondependent lung to be at least partially inflated for a period of some minutes.

\section{Selective dependent lung PEEP}

Since the ventilated lung often has a decreased lung volume during one-lung ventilation, several attempts have been made to improve $\mathrm{PaO}_{2}$ by treating 
the ventilated lung with PEEP. However, although selective dependent lung PEEP may improve ViQ relationships in the dependent lung, it may also increase dependent lung PVR and shunt blood flow to the nonventilated nondependent lung causing no change or even a decrease in $\mathrm{PaO}_{2}$ (Figure 8 , right upper panel).

\section{Selective nondependent lung CPAP}

PEEP can be selectively applied to just the nonventilated nondependent lung. Under these conditions the nonventilated lung is only slightly but constantly distended by oxygen, so that a better term for this ventilatory pattern arrangement would be nonventilated lung continuous positive airway pressure (CPAP). Recently, two reports, one in humans ${ }^{5}$ and one in dogs, ${ }^{6}$ have shown that the application of CPAP to the nonventilated lung increased $\mathrm{PaO}_{2}$ significantly. The latter study was performed with the dogs in the lateral decubitus position and showed that low levels of CPAP $\left(5-10 \mathrm{~cm} \mathrm{H}_{2} \mathrm{O}\right)$ to the nonventilated lung increased $\mathrm{PaO}_{2}$ and decreased shunt, while blood flow to the nonventilated lung remained unchanged. Therefore, low levels of CPAP simply maintained the patency of nondependent lung airways allowing some oxygen distention of the gas exchanging alveolar space in the nondependent lung (Figure 8, lower left panel). On the other hand, $15 \mathrm{~cm} \mathrm{H}_{2} \mathrm{O}$ of CPAP caused similar $\mathrm{PaO}_{2}$ and shunt changes while blood flow to the nonventilted lung decreased significantly. Therefore, high levels of nonventilated lung CPAP act by permitting oxygen uptake in the nonventilated lung as well as by causing blood flow diversion to the ventilated lung where both oxygen and carbon dioxide exchange can take place (Figure 2, lower left panel). Since low levels are as effective as high levels of nonventilated lung CPAP and have less surgical interference and haemodynamic implications, it is logical to use low levels of nonventilated CPAP first. Low levels of nonventilated CPAP usually correct severe hypoxaemia $\left(\mathrm{PaO}_{2}<50\right.$ $\mathrm{mmHg}$ ) provided that the DLT is positioned correctly. In both the human and dog studies, $\mathrm{O}_{2}$ insufflation at zero airway pressure did not improve $\mathrm{PaO}_{2}$ and shunt significantly, and this was probably due to the inability of zero transbronchial airway pressure to maintain airway patency. Several easyto-assemble selective nondependent lung CPAP systems have been described recently.

\section{Differential lung PEEP, CPAP}

From the above considerations, it seems that the ideal method to improve $\mathrm{PaO}_{2}$ during one-lung ventilation is the application of differential lung PEEP or PEEP/CPAP. In this situation, PEEP is applied to the ventilated lung in the conventional manner to improve ventilated lung volume and $\dot{V} / \dot{Q}$ relationships. Simultaneously, the nonventilated lung receives CPAP to improve oxygenation of the blood perfusing the nonventilated lung. Therefore, with differential lung PEEP or PEEP/CPAP, the distribution of blood flow is not a critical issue because all pulmonary perfusion can participate in oxygen uptake from both oxygen expanded lungs (Figure 8, lower right panel).

\section{Summary of special one-lung management manoeuvres}

The foregoing discussion indicates that the sequence of treating hypoxemia during one-lung ventilation in the lateral decubitus position should be as follows. First, $5-10 \mathrm{~cm} \mathrm{H}_{2} \mathrm{O}$ of CPAP should be applied to the nonventilated nondependent lung. Nondependent lung CPAP should be applied during the deflation phase of a large tidal volume breath to overcome critical opening pressures in the atelectatic lung. If oxygenation still does not improve, then 5-10 $\mathrm{cm} \mathrm{H}_{2} \mathrm{O}$ of PEEP to the ventilated dependent lung should then be applied. Finally, if oxygenation is still not satisfactory, dependent lung PEEP should be increased to $10-15 \mathrm{~cm} \mathrm{H}_{2} \mathrm{O}$ of PEEP while the nondependent lung is maintained at $5-10 \mathrm{~cm} \mathrm{H}_{2} \mathrm{O}$ CPAP. The differential lung CPAP/ PEEP search is conducted in this way to find the optimal end-expiratory pressure for each lung and minimum $\dot{Q}_{s} / \dot{Q}_{t}$ for the patient as a whole. However, in view of the usual efficacy of nonventilated nondependent lung CPAP, use of differential lung PEEP/CPAP should rarely be necessary. If severe hypoxemia is still present following the use of differential lung CPAP/PEEP, it should be remembered that the nondependent lung may be intermittently ventilated with positive pressure with oxygen. Finally, most of the $\dot{V} / \dot{Q}$ imbalance can be eliminated during a pneumonectomy by tightening a ligature around the nonventilated lung pulmonary artery as early as possible which eliminates shunt flow through the nonventilated lung. 


\section{References}

1 Benumof $J L$, Alfery $D D$. Anesthesia for thoracic surgery. In: Miller R (Ed.): Anesthesia 2nd ed. New York: Churchill Livingstone, 1985.

2 Keating $J L$, Benumof $J L$. Analysis of margin of safety in positioning double-lumen tubes. Anesthesiology 1985; 63: A563.

3 Benumof $J L$. One-lung ventilation and hypoxic pulmonary vasoconstriction: implications for anesthetic management. Anesth Analg 1985; 64: 821-33.

4 Benumof JL. Physiology of the open chest and one lung ventilation. In: Kaplan J (Ed.): Thoracic Anesthesia. New York: Churchill Livingstone, 1983.

5 Rogers $S N$, Benumof $J L$. Halothane and isoflurane do not decrease $\mathrm{PaO}_{2}$ during one-lung ventilation in intravenously anesthetized patients. Anesth Analg 1985; 64: 946-54.

6 Augustine $S D$, Benumof $J L$. Halothane and isoflurane do not impair arterial oxygenation during one-lung ventilation in patients undergoing thoractomy. Anesthesiology 1984; 61: A484.

7 Benumof $J L$. One-lung ventilation: which lung should be PEEPed? (Editorial). Anesthesiology 1982; 56: 161-3.

8 Capan LM, Turndorf H, Patel C, Ramanathan $S$, Acinapura A, Chalon J. Optimization of arterial oxygenation during one-lung anesthesia. Anesth Analg 1980; 59: 847-51.

9 Alfery $D D$, Benumof $J L$. Improving oxygenation during one-lung ventilation in dogs: the effects of PEEP and blood flow restriction to the nonventilated lung. Anesthesiology 1981; 55: 381-5. 